\title{
Isolation and characterization of bacteria from diabetic foot ulcer: amputation, antibiotic resistance and mortality rate
}

\author{
Muamar M. A. Shaheen ${ }^{1}$ (D) Sewar Al Dahab ${ }^{1} \cdot$ Maryiam Abu Fada $^{1} \cdot$ Rawand Idieis $^{1}$
}

Received: 20 February 2021 / Accepted: 23 August 2021 / Published online: 10 September 2021

(c) Research Society for Study of Diabetes in India 2021

\begin{abstract}
Background Diabetic foot ulcer (DFU) is one of the most serious complications of diabetes mellitus with devastating outcomes. Poorly treated DFU leads to osteomyelitis, gangrene and limb amputation. There is an increased risk of mortality for the amputees and increased number of bacterial resistance in survived patients. Struggle on choice of the best antibiotic(s) for DFU is escalating.

Objectives To determine risk factors associated with mortality in patients with DFU. To investigate bacterial drug resistance in survived or deceased patients around amputation.

Methodology This is a retrospective cohort study that involved all diabetic patients who had DFU or minor or major amputation at Hebron Governmental Hospital from 2013 to 2020. Antibiotic use and bacterial isolates along with culture and sensitivity test results were retrieved from patients' profiles and laboratory records. Major outcome of study was survival rate around amputation. Patients who missed test results for FBS or HbAc1, or who had no wound culture were excluded. SPSS version 22 was used to analyze data.

Results Eighty four subjects were included in this study, $64.8 \pm 12.58$ years old, $63.1 \%$ males who had diabetic foot ulcer, minor or major limb amputation between 2013 and 2020 at Hebron Governmental Hospital. Forty tow patients (50\%) had diabetic foot ulcer, 28 patients (33.3\%) had major limb amputation, and 14 patients (16.7\%), succumbed to minor amputation. Average FBS was $292.8 \pm 136.33 \mathrm{mg} / \mathrm{dl}$ and average $\mathrm{HbA1C}$ was $8.55 \pm 1.89 \%$. Mortality rate was $9.5 \%$. Using the Chi square test, we found a significant relationship between mortality and type of isolated bacteria, $p=0.033$ and between diabetic complications (nephropathy) and mortality, $p=0.033$. There was a significant relationship between antibiotic use and mortality, $p=0.04$, especially with metronidazole and colistin, if they were used around limb amputation.
\end{abstract}

Conclusions Mortality of diabetic patients with DFU was associated with nephropathy and Acinetobacter or $E$. coli infections.

Keywords DFU $\cdot$ Amputation $\cdot$ Mortality rate $\cdot$ Antibiotic-resistance $\cdot$ Bacterial isolates

\section{Introduction}

Diabetes mellitus (DM) is a chronic multifactorial condition. It occurs when the pancreas is unable to produce enough insulin or the human body is incapable of using effectively the secreted insulin. Patients with DM suffer a variety of complications due to micro- and macro-vascular abnormalities. Complications such as neuropathy, nephropathy, obesity, hypertension, and increased susceptibility to a variety

Muamar M. A. Shaheen

Muamarsh@hebron.edu

1 Clinical Pharmacy and Practice, Faculty of Pharmacy and Medical Sciences, Hebron University, P.O Box 40, Hebron, West Bank 00972, Palestine of infectious diseases are most common. However, poor control over blood sugar leads to life-threatening complications such as cardiovascular disease, foot damage, hearing impairment, fungal and bacterial infection, Alzheimer disease, memory deficits and depression [1].

Type I DM affects $5-10 \%$ of patients and commonly occurs in young ages which have autoimmune destruction of beta-cell. This type is treated mainly by insulin. Patients in this type are non-obese [2].

Type II DM affects $90 \%$ of patients and commonly occurs in older people which have low sensitivity to insulin. This type of DM is treated by diet. If not well-controlled by diet alone, oral hypoglycemic agent is added to the regimen, then, insulin is added as per case. Nutritional 
medical treatment is another option. Patients in this type are obese and highly affected by family history of DM [3].

Patient education is crucial for all diabetic patients especially those with high risk of DM. High risk patients should be advised to make dietary adjustments, lose excess weight, do regular light exercise, check their blood sugar periodically, smoking cessation, and foot care.

A Palestinian study performed in 2019 demonstrated that smoking, sensory loss to vibration, sensory loss to monofilament, loss of pedal pulse, presence of calluses, nephropathy, retinopathy, neuropathy, and poor self-care behaviors were associated with DFU [4].

The same study above recommended that: knowing risk factors and predictors of amputation will help nurses and physicians design a suitable program to minimize the incidence of DFU development such as integrating audiovisual teaching strategies. Also, increasing public awareness and knowledge about risk factors and importance of self-care practices by the healthcare team can decrease the incidence of diabetic foot ulcers in Palestine.

One of the most serious complications of DM is diabetic foot ulcer (DFU). It occurs more commonly in males than females as suggested by a recent study in Saudi Arabia. Up to $2-3 \%$ of patients with DM are thought to have an active foot ulcer, it is also increasingly recognized that latter stages of complications from foot ulcers are associated with serious morbidity and overall reduction in quality of life. Managing tissue damage and enhancing wound healing and repair in DFU might be quite challenging. Wound dressings that might form a barrier against contamination and debridement by removal of necrotic and hyperkeratotic tissue are widely used. Improving vascularization through Percutaneous Trans luminal angioplasty (PCTA) is the gold standard for placement of narrow blood vessels with or without stents. This procedure can also improve blood flow and promote healing in a timely manner. It is estimated that more than two-thirds of non-traumatic lower limb amputations are preceded by an ulcer $[5,6]$.

Foot ulcer commonly affects people with type II DM. It can lead to infection and amputation of lower extremities. The risk of developing an ulcer increases with peripheral vascular disease, neuropathy, diabetes duration $\geq 10$ years, insulin use, retinopathy, nephropathy, age 45 years, cerebral vascular disease, and poor glycemic control. Increasing cumulative glycemic burden, coronary artery disease, male gender, smoking, and hypertension are all present in these patients [7-10].

Patients with poorly treated DFU may develop diabetic foot osteomyelitis leading to gangrene and amputation. Diabetic foot infections (DFI) are predominantly polymicrobial and multidrug resistant (MDR) with the ability to form biofilm [11].
Our study aims at determining main risk factors associated with lower limb amputation among diabetic patients in Palestine. We focused on survival rate and quality of life of patients after amputation. This issue was poorly studied in the literature.

We aim at tracing antibiotic resistance among non-hospitalized patients with recurrent infection and re-hospitalization at time of severe infection and amputation.

\section{Methods}

We decided to review all diabetic patients' profiles with DFU who went through foot, part of foot, or below knee amputation at the orthopedic department at Hebron Governmental Hospital between 2013 and 2020. We defined 126 patients who complied with our inclusion criteria. Unfortunately, we had to exclude some of them due to missing information such as FBS or HbAc1, bacterial culture and antibiotic sensitivity test results around amputations. Sensitivity test was done using fully automated Mcfarland antibiotic sensitivity test using VITEX 2 compact device, without Mueller Hinton agar. Bacterial swabs were taken from all DFU patients and bacterial identification by culture was done. SPSS version 22 was used for analysis of data.

\section{Results}

A total of 84 patients with diabetic foot infection were included in the present study whose sociodemographic and clinical data are shown in Table 1 below. The mean age of patients was $64.8 \pm 12.58$ with predominantly male patients $53(63.1 \%)$. Majority of subjects (95.24\%) had Type 2 diabetes mellitus. Mean $\mathrm{HbA1c}$ of the patients was $8.55 \pm 1.89 \%$ and mean FBS was $292.8 \pm 136.33 \mathrm{mg} / \mathrm{dl}$ (Table 1).

Patients were then categorized according to main outcome of study, the survival rate. Bacterial isolates from survived or dead patient's foot were identified as shown in Table 2.

Chi square test showed a significant relation between death and microorganism isolated from diabetic foot, $p=0.033$. The results also indicated a significant relation between diabetic complications and death, $p=0.033$, Table 2.

We also depicted percentage of bacteria species isolated from diabetic foot as related to main outcomes of the study as shown in Fig. 1.

However, when we stratified bacterial species with diabetic complications, we found that all dead patients suffered from some kind of diabetic complications. For example, $100 \%$ of dead patients who had Proteus, Enterococcus or Enterobater species isolates, had nephropathy. 
Table 1 Sociodemographic, diabetic complications, microorganism isolated and antibiotic use per diagnosis

\begin{tabular}{|c|c|c|c|}
\hline \multicolumn{4}{|l|}{ Diagnosis } \\
\hline Variables & $\begin{array}{l}\text { Diabetic foot ulcer } \\
N(\%)\end{array}$ & $\begin{array}{l}\text { Major amputation } \\
\mathrm{N}(\%)\end{array}$ & $\begin{array}{l}\text { Minor amputation } \\
\mathrm{N}(\%)\end{array}$ \\
\hline \multicolumn{4}{|l|}{ Microorganism } \\
\hline Proteus spp. & $9(21.4)$ & $3(21.4)$ & $5(17.9)$ \\
\hline Klebsiella pneumoniae & $5(11.9)$ & $1(7.1)$ & $9(32.1)$ \\
\hline Stappylococcus aureas & $5(11.9)$ & $2(14.3)$ & $3(10.7)$ \\
\hline Pseudomonas spp. & $3(7.1)$ & $1(7.1)$ & $2(7.1)$ \\
\hline MRSA & $8(19)$ & $2(14.3)$ & $3(10.7)$ \\
\hline Acinetobacter spp. & $4(9.5)$ & $3(21.4)$ & $1(3.6)$ \\
\hline${ }^{\ddagger}$ E. coli & $4(9.5)$ & $1(7.1)$ & $4(14.3)$ \\
\hline Enterococcus spp. & $1(2.4)$ & $1(7.1)$ & $1(3.6)$ \\
\hline Enterobacter spp. & $2(4.8)$ & $0(0)$ & $0(0)$ \\
\hline Staphylococcus epidermidis & $1(2.4)$ & $0(0)$ & $0(0)$ \\
\hline Total & $42(100)$ & $14(100)$ & $28(100)$ \\
\hline \multicolumn{4}{|l|}{ Complication } \\
\hline Nephropathy & $3(7.1)$ & $3(21.4)$ & $3(10.7)$ \\
\hline Retinopathy & $2(4.8)$ & $1(7.1)$ & $3(10.7)$ \\
\hline Cardiovascular problem & $2(4.8)$ & $1(7.1)$ & $1(3.6)$ \\
\hline Peripheral vascular disease & $4(9.5)$ & $1(7.1)$ & $8(28.6)$ \\
\hline Neuropathy & $31(73.8)$ & $8(57.1)$ & $13(46.4)$ \\
\hline Total & $42(100)$ & $14(100)$ & $28(100)$ \\
\hline \multicolumn{4}{|l|}{ D.M ${ }^{*}$} \\
\hline Type 1 & $2(4.8)$ & $0(0)$ & $2(7.1)$ \\
\hline Type 2 & $40(95.2)$ & $14(100)$ & $26(92.9)$ \\
\hline Total & $42(100)$ & $14(100)$ & $28(100)$ \\
\hline \multicolumn{4}{|l|}{ Antibiotic } \\
\hline Ceftriaxon & $16(38.1)$ & $2(14.3)$ & $8(28.6)$ \\
\hline Ceftazidime & $3(7.1)$ & $0(0)$ & $2(7.1)$ \\
\hline Meropenem & $6(14.3)$ & $3(21.4)$ & $3(10.7)$ \\
\hline Colistin & $4(9.5)$ & $3(21.4)$ & $1(3.6)$ \\
\hline Teicoplanin & $1(2.4)$ & $3(21.4)$ & $2(7.1)$ \\
\hline Gentamicine & $2(4.8)$ & $0(0)$ & $0(0)$ \\
\hline Cefuroxime & $(9.5)$ & $3(21.4)$ & $3(10.7)$ \\
\hline Amoxicillin/clavulanic acid & $1(2.4)$ & $0(0)$ & $2(7.1)$ \\
\hline Metronidazole & $4(9.5)$ & $0(0)$ & $3(10.7)$ \\
\hline Vancomycin & $1(2.4)$ & $0(0)$ & $1(3.6)$ \\
\hline Ciprofloxacin & $0(0)$ & $0(0)$ & $3(10.7)$ \\
\hline Total & $42(100)$ & $14(100)$ & $28(100)$ \\
\hline \multicolumn{4}{|l|}{$\mathrm{FBG}^{\S}$} \\
\hline $70-110$ & $0(0)$ & $2(15.4)$ & $0(0)$ \\
\hline $111-126$ & $3(8.1)$ & $0(0)$ & $2(8.7)$ \\
\hline 127-199 & $9(24.3)$ & $3(23.1)$ & $4(17.4)$ \\
\hline More than 200 & $25(67.6)$ & $8(61.5)$ & $17(73.9)$ \\
\hline Total & $42(100)$ & $14(100)$ & $28(100)$ \\
\hline \multicolumn{4}{|l|}{ Age } \\
\hline $30-45$ & $1(2.4)$ & $0(0)$ & $0(0)$ \\
\hline $46-61$ & $15(35.7)$ & $5(35.7)$ & $11(39.3)$ \\
\hline $62-77$ & $20(47.6)$ & $5(35.7)$ & $13(46.4)$ \\
\hline $78-93$ & $6(14.3)$ & $4(28.6)$ & $4(14.3)$ \\
\hline Total & $42(100)$ & $14(100)$ & $28(100)$ \\
\hline
\end{tabular}


Table 1 (continued)

\begin{tabular}{llll}
\hline Diagnosis & & & \\
\hline Variables & $\begin{array}{l}\text { Diabetic foot ulcer } \\
\mathrm{N}(\%)\end{array}$ & $\begin{array}{l}\text { Major amputation } \\
\mathrm{N}(\%)\end{array}$ & $\begin{array}{l}\text { Minor amputation } \\
\mathrm{N}(\%)\end{array}$ \\
\hline Mortality & & & \\
Survived & $36(85.7)$ & $14(100)$ & $26(29.9)$ \\
Deceased & $6(14.3)$ & $0(0)$ & $2(7.1)$ \\
Total & $42(100)$ & $14(100)$ & $28(100)$ \\
\hline
\end{tabular}

E. coli, Escherichia coli; ${ }^{\ddagger} D . M$, Diabetes Mellitus; ${ }^{\S} F B G$, Fasting Blood Glucose, $\mathrm{mg} / \mathrm{dl}$

Table 2 Percentage survival rates per microorganisms isolated or diabetes mellitus complications at time of amputation

\begin{tabular}{lllll}
\hline & $\begin{array}{l}\text { Survived } \\
\mathrm{N}(\%)\end{array}$ & $\begin{array}{l}\text { Dead } \\
\mathrm{N}(\%)\end{array}$ & Chi-square statistic & Sig \\
\hline Microorganism & & & & \\
Proteus spp. & $16(21.1)$ & $1(12.5)$ & 14.016 & \\
Klebsiella pneumoniae & $15(19.7)$ & $0(0)$ & \\
Staphylococcus aureas & $10(13.2)$ & $0(0)$ & \\
Pseudomonas spp.* & $5(6.6)$ & $1(12.5)$ & \\
MRSA & $13(17.1)$ & $0(0)$ & \\
Acinetobacter spp. & $6(7.9)$ & $2(25)$ & \\
E. coli & $7(9.2)$ & $2(25)$ & \\
Enterococcus spp. & $2(2.6)$ & $1(12.5)$ & \\
Enterobacter spp. & $1(1.3)$ & $1(12.5)$ & \\
Staphylococcus epidermidis & $1(1.3)$ & $0(0)$ & \\
Total & $76(100)$ & $8(100)$ & \\
Complications & & & \\
Nephropathy & $5(6.6)$ & $4(50)$ & 14.016 \\
Retinopathy & $6(7.9)$ & $0(0)$ & \\
Cardiovascular problem & $4(5.3)$ & $0(0)$ & \\
Peripheral vascular disease & $11(14.5)$ & $2(25)$ & \\
Neuropathy & $50(65.8)$ & $2(25)$ & \\
Total & $76(100)$ & $8(100)$ & \\
\hline
\end{tabular}

${ }^{\ddagger} M R S$, Methicillin Resistant Staphylococcus aureas; ${ }^{* *}$ spp., species
On the other hand, Pseudomonas species isolates along with neuropathy lead to death for all patients, as shown in Fig. 2.

We also sought antibiotic percentage use among dead and survived subjects to provide a clear picture of antibiotic contribution to the main outcome. As shown in Fig. 3, high percentage usage of certain antibiotic was found in dead subjects around amputation, such as metronidazole.

We followed the trends of antibiotic use and bacterial resistance over the past 7 years (2013-2020). There was inconsistent use of antibiotics through these years for many reasons such as; antibiotic availability, appearance of specific antibiotic-resistant bacteria, and protocol and clinical decision.

We could find sufficient data for the past 5 years only about trends of antibiotic use and bacterial isolate, see Fig. 4. Empty year columns mean no use of that antibiotic at that year due to emergence of resistance as was confirmed by bacterial culture and sensitivity test. (Only consistently used antibiotics with confirmed cultures and sensitivity tests during these years were shown here. You can follow by color any antibiotic through all years or any bacteria per antibiotic per year in this figure.)

\section{Discussion}

Many studies focused on microorganism species isolated from diabetic foot without linking these findings to limb amputation or survival rates among afflicted patients. Here are some of these studies. Kaimkhani et al. found that the most common organisms isolated from DFU are Staphylococcus aureus, Proteus, Pseudomonas, and Escherichia coli [12]. 
Fig. 1 Percentage of bacterial isolates from subjects before death or in survived patients around amputation

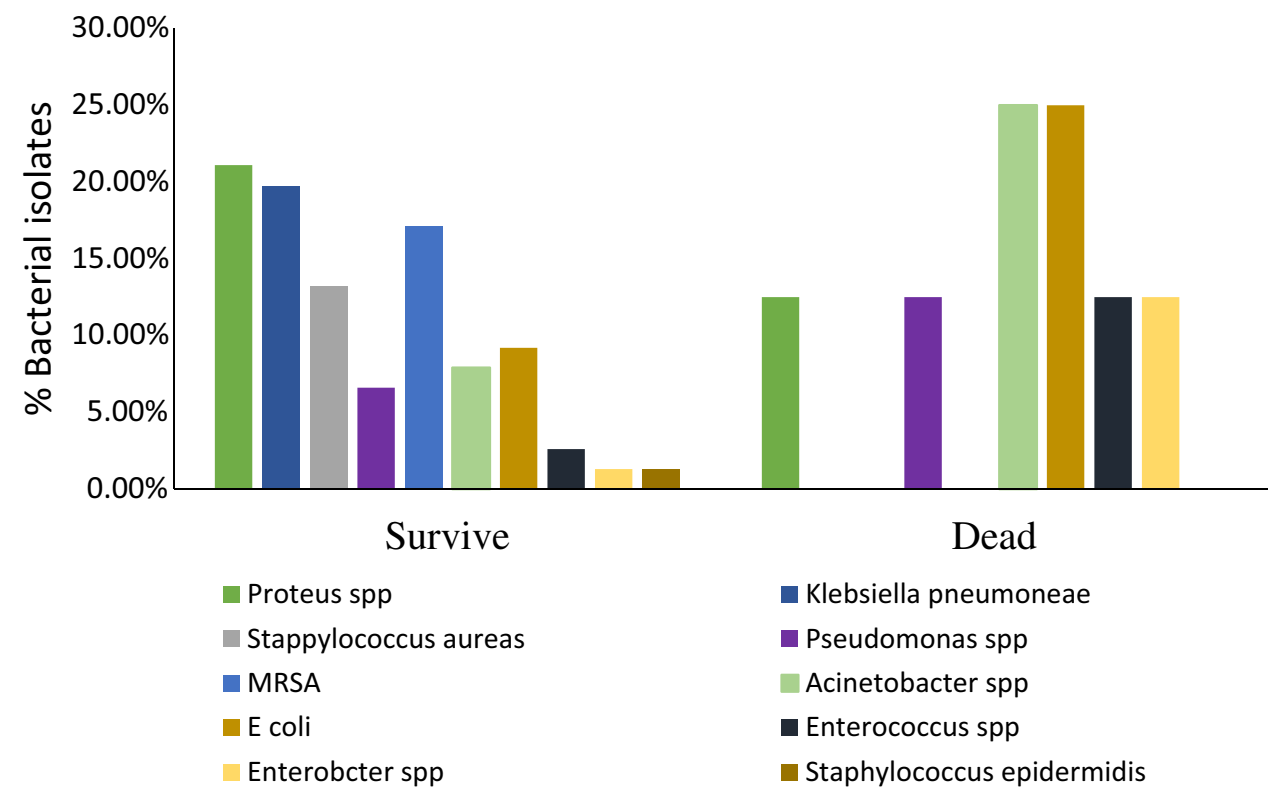

Fig. 2 Percentage of diabetic complications and type of bacteria isolated from foot of dead subjects at time of amputation

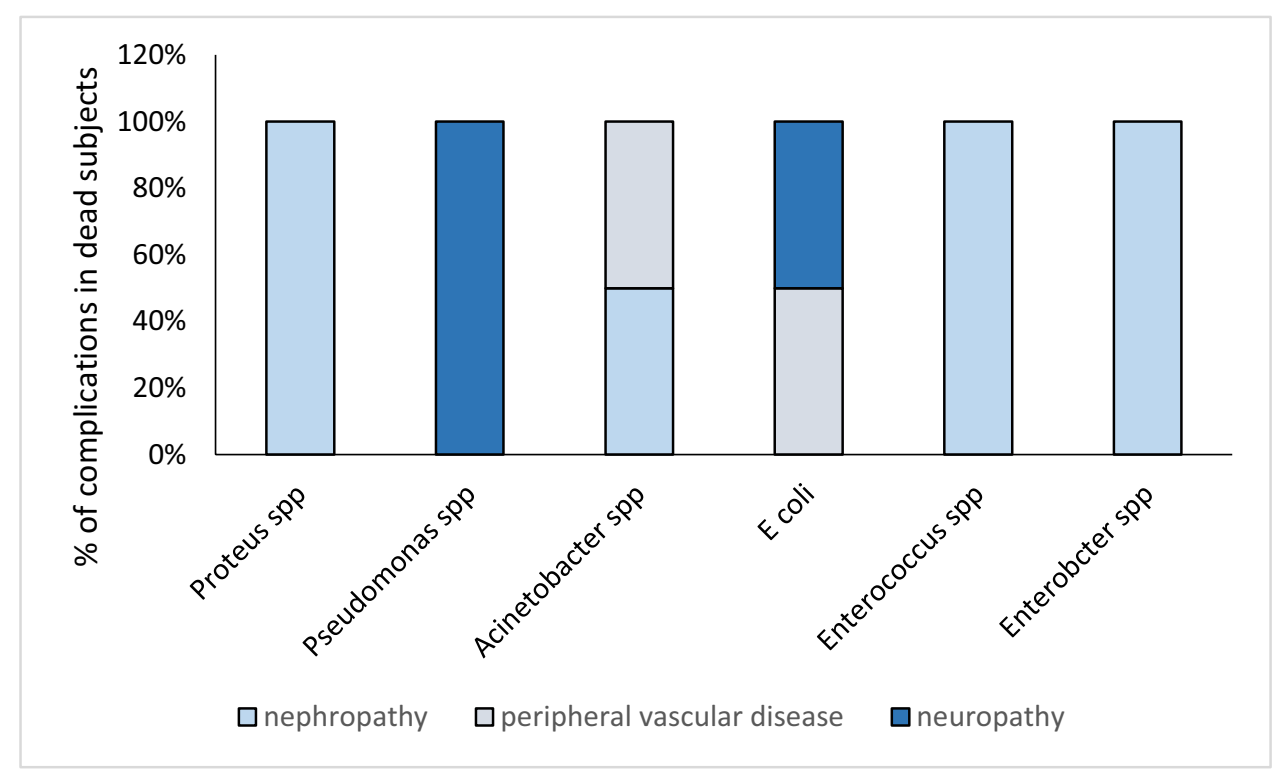

Carro et al. (2019) found that gram negative bacteria were the most frequent microorganisms isolated from DFU and the study recommended an empiric therapy of antibiotics with amoxicillin/clavulanate plus ciprofloxacin should be given as a regimen of choice for management [13].

In Lebanon, a study found that Pseudomonas spp. was the most common Gram-negative organism isolated from DFU in some Middle Eastern countries. The study also showed that a combination of amoxicillin/clavulanate and ciprofloxacin was the most appropriate empirical oral antibiotic for outpatient. Piperacillin/tazobactam would then be the treatment of choice for hospitalized a patient if oral treatment has failed [14].
In another study that was conducted in Kenya, the majority of organisms isolated were gram negative bacteria. Most common were S. aureus, E. coli, Proteus mirabilis, Klebsiella pneumoniae and Pseudomonas aeruginosa. These bacteria showed resistance to commonly used antibiotics like ampicillin, amoxicillin, ceftazidime and pipracillin with tazobactam [15].

In our study, gram negative bacteria were also the most common bacteria isolated from diabetic foot, such as Proteus species (17 patients), Klebsiella (15 patients), and MRSA (gram positive) (13 patients), among others such as $S$. aureus, E. coli, and Acinobacter species. This comes in align with some studies as above. However, subjects in our 
Fig. 3 Percentage of antibiotics use among patients at time of amputation as related to main outcome of the study

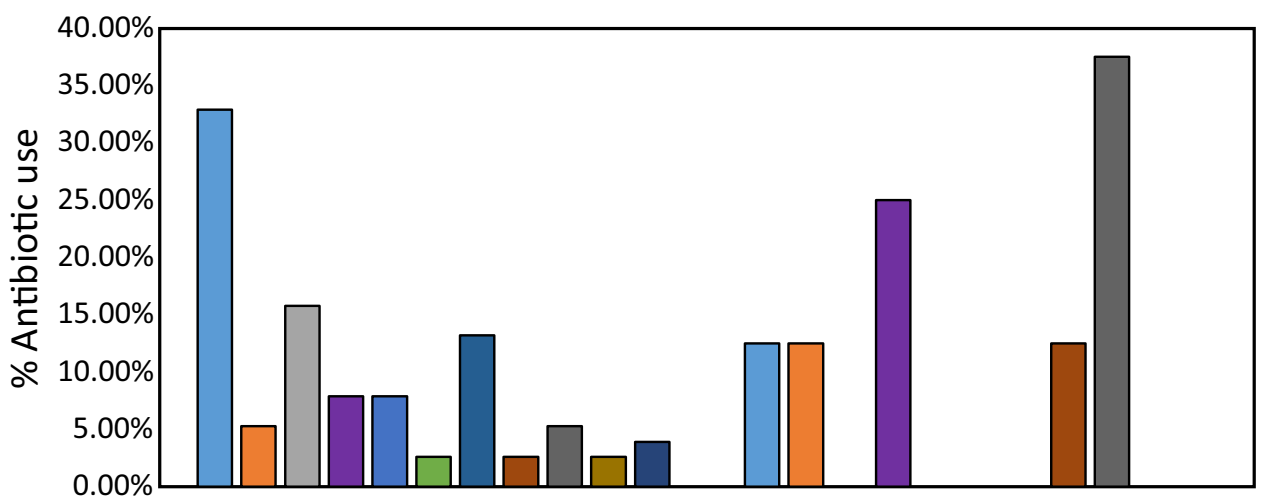

Survive

Dead

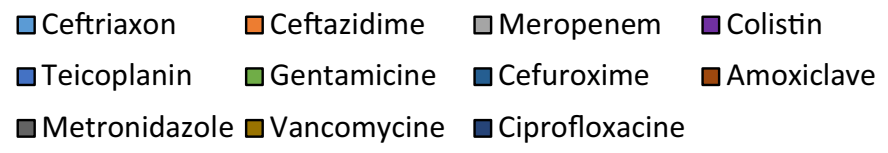

Fig. 4 Bacterial isolates form diabetic foot and antibioticresistance among all subjects of the study during the past 5 years

study have higher percentage of MRSA which is usually hospital-acquired infection that is hard to eradicate.

Our study showed an extensive use of strong antibiotics by parental route (IV or IM) in hospital setting. As shown in Figs. 3 and 4 above, $2^{\text {nd }}, 3^{\text {rd }}$, and $4^{\text {th }}$ generation cephalosporins are extensively used and still showing activity. Almost $34 \%$ of survived patients were on cefuroxime IM around amputation and $37 \%$ of dead subjects were on metronidazole (Fig. 3). Colistin, metronidazole, and amoxicillin/clavulanic acid are facing higher rates of bacterial resistance and their use is increasingly associated with death. This is similar to the results of Kenya and Lebanon studies above.

Mixed infections (bacterial with fungal) are rare but also found in DFU patients. Candida species (albicans and parapsilosis) and Aspergillus flavus were the most common fungal isolates from DFU. They were successfully treated with oral fluconazol. Seemingly, another study showed that candida was resistant to fluconazol and Aspergillus was resistant to itraconazole. Fungal infection if not recognized and treated can impede wound healing [16-18]. Minority of cases in our 
study had mixed infection and were treated successfully but were not included in the study.

A plethora of studies addressed the risk factors associated with DF amputation whether it was major amputation (above the ankle) and minor amputation (below the ankle). Major amputations were associated with short recovery time and greater mortality rate. The rate of amputation was directly proportional to $\mathrm{HbA} 1 \mathrm{c}$ values more or equal to $8 \%$. Risk factors for major amputation include increase in WBC count and ulcer history. Minor amputation risk factors include; increased duration of diabetes, increased WBC count, infection, revascularization history, and decreased postprandial blood sugar. Foot deformity and serum urea were also associated with diabetic lower extremity amputations [19-22].

In this study, almost all patients had uncontrolled diabetes as shown by average $\mathrm{HbAC} 1$ around $8.55 \%$ and average FBS around $292 \mathrm{mg} / \mathrm{dl}$. However, we could not find a significant relation between some of the studied factors including cumulative blood sugar, FBS, and other factors in Table 1 and amputation risk. This could be attributed to 2 factors; first, FBS and $\mathrm{HbAC} 1$ were not different among subjects of our study whether they had minor or major amputation or DFU. All patients (dead or survived) had uncontrolled diabetes with complications at varying degrees upon admission to orthopedic department.

Second, we didn't study severity of DFU infection prospectively, as per University of Texas scale [9], which plays major role in progression of infection into major or minor amputation. However, we found a significant relation between diabetic complications and survival rate of patients after amputation. Diabetic complications are an inevitable end result of uncontrolled diabetes. All dead patients ( 8 patients) had diabetic complications, Table 2.

Nephropathy (10\% of patients) was associated with death of all patients who had this complication along with Proteus species isolates, Fig. 2. Noteworthy, Proteus itself, as independent factor, was not associated with highest number of deaths as shown in Table 2. This proves valid the conclusion that nephropathy plays a major role in death in patients with DFU around amputation due to direct effect on infection complications and intervention with antibiotic efficacy and/or excretion rate. This comes in align with a study by Shojaiefard et al. (2008) that found higher incidence of nephropathy(as independent factor) in patients who needed amputation [23].

Neuropathy (62\%) of patients and peripheral vascular disease $(15 \%)$ were independently associated with death of half patients with $E$. coli isolates, Fig. 2. Other studies related neuropathy and peripheral vascular disease to amputation in DFU but not to death [24, 25].

One might expect that MRSA, a multidrug resistant bacteria, should lead to death in rates more than E. coli or other species. This was not the case in our study since patients who had MRSA infection didn't have diabetic complications as shown in Fig. 2 above.

However, Acinobacter species and E. coli were associated with highest percentage of death in our patients around amputation. Antibiotic-resistance might be one of the contributing factors and the deterioration of patient condition might be the main reason. This is an epidemiologic evidence of increasing death rate with these bacterial species. We can't really confirm causative relationship with death, Table 2. We didn't confirm direct cause of death among these patients neither did we calculate time elapsed between amputation and death. This might be one of the limitations of this study.

A study by Martins-Mendes, et al., found that complication count and previous DFU were related with death [26]. They also concluded that previous DFU was associated with DFU, amputation and death. Actually, many of our patients had recurrent DFU infections and went through serial amputations before final major amputation with or without death.

Our study doesn't present a solution for antibiotic resistance neither gives suggestions for antibiotic of choice for use, since there isn 't one antibiotic or a combination of antibiotics that might fit all patents at all times. This came along with other studies conclusions where it was found that no single antibiotic used empirically offers adequate coverage for all potential bacteria found in diabetic foot ulcers [27]. In addition to that, poor penetration of antibiotics into the lower limb tissue due to peripheral arterial disease makes therapy less effective [28].

Thirteen of our patients (15\%) (11 survived after amputation and 2 dead) had peripheral vascular disease as explained above.

It was so frustrating to see the extensive use of parenteral antibiotics in these patients to the degree they are no longer effective and patients are losing their choices. Actually patients and their families or care-providers share responsibility in that. We have noticed that subjects in this study came to hospital at end stage of infection with very poor control of blood sugar. Both factors lead to devastating end of amputation.

To make sense of all what we have done, we followed trends of antibiotic use along with bacterial resistance as was confirmed by bacterial culture and sensitivity tests during the past years in this hospital. It was shown that $E$. coli, which happens to be sensitive to many agents in the year 2015, was no longer sensitive to any of them in 2016, as shown in Fig. 4 above. On the other hand, Klebsiella species that were sensitive to many of the listed agents in 2017, were no longer sensitive to any of them in 2018. S. aureus became resistant to almost all listed drugs in 2020 including cefotaxime that was effective in 2018-2019. MRSA was found to be resistant to most listed drugs since 2019 . 
Management of DFU firstly can be achieved by good glycemic control. Pharmacological therapy using suitable antibiotics depending on wound culture result comes next. Quite few studies suggested that intake of some vitamins improves wound healing. These vitamins include; magnesium, zinc and vitamin D. Significant improvement in DFU was observed when these vitamins were taken for 12 weeks [29-31].

Patients with DFUs and COVID-19 infection who require surgical intervention should be treated with considerable care. The surgery should be performed under protective conditions in a negative-pressure operating room. General anesthesia should be avoided in these patients [32].

We highly recommend early diagnosis and treatment of DFU, routine foot inspection, training and employment of Podiatricians in all diabetic clinics of the ministry. Also early use of pressure off-loading devices might protect patients' foot and prevent amputation or worsening of bacterial infection.

We have noticed that amoxicillin/clavulanate and/or ciprofloxacin were the drugs of choice for empirical treatment in most patients in this hospital as part of the general protocol of management by the ministry. However, as we found in our study later, most bacterial species are resistant now to both agents and to combination of both. We recommend highly the personal approach of management of DFU and that there is no single antibiotic that fits all patients. On the light of that, we refused to accept the use of antibiotics upon availability or as per the hospital formulary or best list. The use of IV, or IM antibiotics should be limited to severe cases and for few days only, then a patient has to step down to oral treatment in order to minimize kidney failure and antibiotic resistance. In addition to that, we recommend exploring the possibility of Ozone clinics in the country.

\section{Study limitations}

We didn't determine the survival rate of patients per infection severity neither did we classify bacterial infection into aerobic or anaerobic bacteria, which could shed light on important aspects of resistance and severity of infection.

We focused on sensitivity of bacteria to antibiotic(s) used around amputation without linking it to severity of DFI according to Wagnar or other universal systems due to the retrospective nature of the study and availability of information in the profiles.

Year of survival after amputations was not determined, since it wasn't one of our end results to determine survival rate, rather than death or amputation as the main outcomes.

Small sample size makes it hard to globally generalize conclusions from this study; however, results might be valid in the Middle East.

\section{Conclusions}

Mortality rate is alarmingly increasing in diabetic patients who suffer from diabetic foot ulcer with or without amputation. Mortality was associated with nephropathy and Acinetobacter species or $E$. coli infections. Wise use of antibiotics and personalized treatment of DFU should be adopted.

Acknowledgements We would like to thank the staff at Hebron Governmental Hospital (Formerly Queen Alia Hospital) especially the laboratory department, attending physicians in the orthopaedic department, and pharmacy department for their cooperation with our team in order to get this work done.

\section{Declarations}

Ethics approval and consent to participate First, we took the permission to run this study from the ethics committee at Hebron University (IRB). Then we applied to the Ministry of Health, department of research and continuous learning to get their permission in order to get access to patients ' profiles at the intended hospital. After we got the approval from the minister of health, we approached the hospital administration board and president in order to start the work.

Conflict of interest We declare no conflict of interest for this research.

\section{References}

1. Kim H-G. Cognitive dysfunctions in individuals with diabetes mellitus. Yeungnam Univ J Med. 2019;36(3):183.

2. Mellitus D. Diagnosis and classification of diabetes mellitus. Diabetes Care. 2005;28(S37):S5-10.

3. Serván PR. Diet recomendations in diabetes and obesity. Nutr Hosp. 2018;35(Spec No4):109-15.

4. Salameh BS, Abdallah J, Naerat EO. Case-Control study of risk factors and self-care behaviors of foot ulceration in diabetic patients attending primary healthcare services in Palestine. J Diabetes Res. 2020;2020.

5. Lim JZM, Ng NSL, Thomas C. Prevention and treatment of diabetic foot ulcers. J R Soc Med. 2017;110(3):104-9.

6. Qari FA, Akbar D. Presentation and treatment. Saudi Med J. 2000;21(5):443-6.

7. Wouter B, Abu-Hanna A, Bus SA. Development of a multivariable prediction model for plantar foot ulcer recurrence in high-risk people with diabetes. BMJ Open Diabetes Res Care. 2020;8(1):e001207.

8. Tolossa T, Mengist B, Mulisa D, Fetensa G, Turi E, Abajobir A. Prevalence and associated factors of foot ulcer among diabetic patients in Ethiopia: a systematic review and meta-analysis. BMC Public Health. 2020;20(1):1-14.

9. Smith-Strøm H, Iversen MM, Igland J, Østbye T, Graue M, Skeie $\mathrm{S}$, et al. Severity and duration of diabetic foot ulcer (DFU) before seeking care as predictors of healing time: a retrospective cohort study. PLoS One. 2017;12(5):e0177176.

10. Dekker RG, Qin C, Ho BS, Kadakia AR. The effect of cumulative glycemic burden on the incidence of diabetic foot disease. J Orthop Surg Res. 2016;11(1):1-8.

11. Banu A, Hassan MMN, Rajkumar J, Srinivasa S. Spectrum of bacteria associated with diabetic foot ulcer and biofilm formation: a prospective study. Australas Med J. 2015;8(9):280. 
12. Kaimkhani GM, Siddiqui AA, Rasheed N, Rajput MI, Kumar J, $\mathrm{Khan} \mathrm{MH}$, et al. Pattern of infecting microorganisms and their susceptibility to antimicrobial drugs in patients with diabetic foot infections in a tertiary care hospital in Karachi, Pakistan. Cureus. 2018;10(6).

13. Carro GV, Carlucci E, Priore G, Gette F, Llanos MDLÁ, Losada $\mathrm{D}$, et al. Infecciones en pie diabético. Elección del tratamiento antibiótico empírico. Medicina (Buenos Aires). 2019;79(3).

14. Obeid M, Moughames E, Aboulhosn P, Madi R, Farah M, Feghali $\mathrm{J}$, et al. Epidemiology and susceptibility profiles of diabetic foot infections in five hospitals in Lebanon. J Infect Dev Ctries. 2018;12(05):347-51.

15. Mutonga DM, Mureithi MW, Ngugi NN, Otieno FC. Bacterial isolation and antibiotic susceptibility from diabetic foot ulcers in Kenya using microbiological tests and comparison with RTPCR in detection of S. aureus and MRSA. BMC Res Notes. 2019;12(1):1-6.

16. Torrence GM, Schmidt BM. Fungal osteomyelitis in diabetic foot infections: a case series and comparative analysis. Int J Low Extrem Wounds. 2018;17(3):184-9.

17. Raiesi O, Siavash M, Mohammadi F, Chabavizadeh J, Mahaki B, Maherolnaghsh M, et al. Frequency of cutaneous fungal infections and azole resistance of the isolates in patients with diabetes mellitus. Adv Biomed Res. 2017;6.

18. Missoni EM, Kalenić S, Vukelić M, De Syo D, Belicza M, Kern $\mathrm{J}$, et al. Role of yeasts in diabetic foot ulcer infection. Acta Med Croat . 2006;60(1):43-50.

19. Atosona A, Larbie C. Prevalence and determinants of diabetic foot ulcers and lower extremity amputations in three selected tertiary hospitals in Ghana. J Diabetes Res. 2019;2019.

20. Jiang Y, Ran X, Jia L, Yang C, Wang P, Ma J, et al. Epidemiology of type 2 diabetic foot problems and predictive factors for amputation in China. Int J Low Extrem Wounds. 2015;14(1):19-27.

21. Armstrong DG, Lipsky BA. Diabetic foot infections: stepwise medical and surgical management. Int Wound $J$. 2004;1(2): 123-32.

22. Jbour A, Jarrah NS, Radaideh AM, Shegem NS, Bader IM, Batieha AM, et al. Prevalence and predictors of diabetic foot syndrome in type 2 diabetes mellitus in Jordan. Saudi Med J. 2003;24(7):761-4.

23. Shojaiefard A, Khorgami Z, Larijani B. Independent risk factors for amputation in diabetic foot. Int J Diabetes Dev Ctries. $2008 ; 28(2): 32$
24. Shin JY, Roh S-G, Sharaf B, Lee N-H. Risk of major limb amputation in diabetic foot ulcer and accompanying disease: a metaanalysis. J Plast Reconstr Aesthet Surg. 2017;70(12):1681-8.

25. Li X, Xiao T, Wang Y, Gu H, Liu Z, Jiang Y, et al. Incidence, risk factors for amputation among patients with diabetic foot ulcer in a Chinese tertiary hospital. Diabetes Res Clin Pract. 2011;93(1):26-30.

26. Martins-Mendes D, Monteiro-Soares M, Boyko EJ, Ribeiro M, Barata P, Lima J, et al. The independent contribution of diabetic foot ulcer on lower extremity amputation and mortality risk. J Diabetes Complicat. 2014;28(5):632-8.

27. Rahim F, Ullah F, Ishfaq M, Afridi AK, ur Rahman S, Rahman $H$. Frequency of common bacteria and their antibiotic sensitivity pattern in diabetics presenting with foot ulcer. J Ayub Med Coll Abbottabad. 2016;28(3):528-33.

28. Datta P, Chander J, Gupta V, Mohi GK, Attri AK. Evaluation of various risk factors associated with multidrug-resistant organisms isolated from diabetic foot ulcer patients. J Lab Phys. 2019;11(1):58.

29. Razzaghi R, Pourbagheri H, Momen-Heravi M, Bahmani F, Shadi J, Soleimani Z, et al. The effects of vitamin D supplementation on wound healing and metabolic status in patients with diabetic foot ulcer: a randomized, double-blind, placebo-controlled trial. J Diabetes Complicat. 2017;31(4):766-72.

30. Razzaghi R, Pidar F, Momen-Heravi M, Bahmani F, Akbari H, Asemi Z. Magnesium supplementation and the effects on wound healing and metabolic status in patients with diabetic foot ulcer: a randomized, double-blind, placebo-controlled trial. Biol Trace Elem Res. 2018;181(2):207-15.

31. Momen-Heravi M, Barahimi E, Razzaghi R, Bahmani F, Gilasi HR, Asemi Z. The effects of zinc supplementation on wound healing and metabolic status in patients with diabetic foot ulcer: a randomized, double-blind, placebo-controlled trial. Wound Repair Regener. 2017;25(3):512-20.

32. Tao F, Tang X, Tao H, Luo Y, Cao H, Xiang W, et al. Surgical treatment of diabetic foot ulcers during the COVID-19 pandemic in China. J Diabetes Complicat. 2020;34(9):107622.

Publisher's note Springer Nature remains neutral with regard to jurisdictional claims in published maps and institutional affiliations. 